\title{
Shock acceleration in partially neutral plasmas
}

\author{
G. MORLINO*, E. AMATO, P. BLASI and D. CAPRIOLI \\ INAF, Osservatorio Astrofisico di Arcetri, \\ L.go E. Fermi-5, Firenze, 50125, Italy \\ *E-mail:morlino@arcetri.astro.it
}

\begin{abstract}
We present the non-linear theory of shock acceleration applied to SNRs expanding into partially neutral plasma. Using this theory we show how the Balmer lines detected from young SNRs can be used to test the efficiency of shocks in the production of cosmic rays. In particular we investigate the effect of charge-exchange between protons and neutral hydrogen occurring in the precursor formed ahead of the shock. In this precursor the CR pressure accelerate the ionized component of the plasma and a relative velocity between protons and neutral hydrogen is established. On the other hand the charge-exchange process tends to equilibrate ions and neutrals resulting in the heating of both components. We show that even when the shock converts only a few per cent of the total bulk kinetic energy into CRs, the heating is efficient enough to produce a detectable broadening of the narrow Balmer lines emitted by the neutral hydrogen.
\end{abstract}

Keywords: Particle acceleration; Supernova Remnant; Interstellar medium.

\section{Introduction}

Supernova remnants (SNRs) are thought to be the primary sources of Galactic cosmic rays (CRs). Diffusive shock acceleration (DSA) occurring around the forward shock is considered the most promising mechanism for the acceleration of particles up to very high energies. In order to explain the flux of CRs observed at the Earth, the shock should convert a fraction $\sim 10-30 \%$ of the plasm kinetic energy into non thermal particles. Indeed, from the theoretical point of view, DSA can be very efficient in producing accelerated particles, but a clear confirmation of this prediction still lacks, even if many circumstantial evidences have been collected (see e.g. Ref. 1). A possible way to investigate the efficiency of CRs production is through the analysis of the Balmer lines associated with shocks of SNRs which propagate in a partially ionized plasma. The hydrogen lines of a Balmer shock consist of two superimposed components: the narrow 
component is emitted by neutral hydrogen after entering the shock front and the broad component by hot protons after undergoing charge exchange with incoming neutral hydrogen atoms. The width of the broad component reflects the proton temperature behind the shock front, while the width of the narrow component provides a direct measurement of the temperature upstream of the shock. ${ }^{2,3}$

We stress that, when the shock is not modified by the presence of CRs, the charge exchange process and the Balmer emission both occur only downstream of the shock. On the other hand, when CRs are accelerated efficiently, the shock structure is modified and a precursor is generated upstream: the CRs pressure slows down and compresses the ionized plasma. Even if the neutral component is not directly affected by the CR pressure, a relative velocity between ions and neutrals is now established. Hence charge exchange can also occur upstream of the shock, resulting in the pre-heating of neutral hydrogen. We do expect two remarkable consequences: 1) the narrow Balmer lines can be emitted also from the upstream region and 2) the typical width of these lines becomes larger with respect to the case without CRs, because the hydrogen temperature increases.

A further consequence of efficient acceleration is that the temperature of the shocked ions downstream is lower with respect to the case with no acceleration. This occurs because a non negligible fraction of the shock kinetic energy is converted into CRs, rather than into thermal energy. As a consequence also the width of the broad Balmer lines is affected, being reduced with respect to the case with no acceleration.

Remarkably all these aspects have been observed in some Balmerdominated shocks:1) from a region of the Tycho remnant, the emission of narrow Balmer lines has been detected upstream of the shock; ${ }^{4} 2$ ) in several SNRs the narrow Balmer lines present a width incompatible with the typical temperature of the interstellar medium $;{ }^{5} 3$ ) in two different cases where the Balmer emission has been detected, i.e. RCW $86^{6}$ and SNR 0509-67.5, ${ }^{7}$ the width of the broad lines led to a downstream temperature which is lower than that estimated form the measurement of the shock proper motion, suggesting that a fraction of the total energy is converted into CRs.

In order to quantify all these effects simultaneously, in this paper we present the solution for the stationary case of DSA when the shock propagates into a partially ionized plasma. We use a semi-analytical method, similar to those used in Ref. 8 , specialized for plane shock geometry. 


\section{Interaction between ions and neutrals in the precursor}

The process of charge exchange can occur in the precursor only if its typical length scale is smaller than the precursor length. The length of the precursor created by CR pressure is of the order of the diffusion length of the particles with maximum energy, i.e.:

$$
L_{\text {prec }} \simeq D\left(E_{\max }\right) / u_{0}=3 \cdot 10^{17} E_{\mathrm{TeV}} B_{\mu G}^{-1} u_{8}^{-1} \mathrm{~cm},
$$

where the maximum energy is expressed in $\mathrm{TeV}$, the magnetic field in $\mu \mathrm{G}$ and the shock speed is $u_{0}=u_{8} 10^{8} \mathrm{~cm} / \mathrm{s}$. We assume that particles diffuse with a Bohm-like diffusion coefficient, $D(p)=(c / 3) r_{L}(p)$, where $r_{L}=p c / e B$ is the Larmor radius and the magnetic field strength is assumed constant in the precursor. Let call $n_{H}$ the neutral hydrogen density, the length scale for a proton to make charge exchange upstream is:

$$
L_{c e}=u_{0} /\left(n_{H} \sigma_{c e}\left(v_{r}\right) v_{r}\right) \simeq 10^{15}\left(u_{0} / v_{r}\right)\left(n_{H} / 1 \mathrm{~cm}^{-3}\right)^{-1} \mathrm{~cm} .
$$

We note that when the relative speed between ions and neutral hydrogen is $v_{r} \lesssim 2000 \mathrm{~km} / \mathrm{s}$, the charge exchange cross section is roughly constant. ${ }^{3}$ Comparing $L_{c e}$ and $L_{p r e c}$ we see that a single proton can charge exchange several times in the precursor before crossing the shock. In this work we neglect the role of ionization because the ionization cross section is much smaller than the charge exchange length when $v_{r} \lesssim 1000 \mathrm{~km} / \mathrm{s}$, but we will defer a more extensive treatment of ionization to a future paper.

\section{Hydrodynamics with charge-exchange}

The behaviour of a system of neutral hydrogen and protons interacting via charge exchange is described by a kinetic equations for each species:

$$
\frac{\partial f_{i}}{\partial t}+v_{x} \frac{\partial f_{i}}{\partial x}=\beta_{i}(x, \mathbf{v}) f_{j}(x, \mathbf{v})-\beta_{j}(x, \mathbf{v}) f_{i}(x, \mathbf{v}),
$$

where $i, j=H, p$ and $f_{i}$ is the distribution function. Eq. (3) is written in one spatial dimension because we are considering plane shocks propagating along the $x$ direction. As a consequence $f_{i}$ is only a function of the position $x$ and of the particle velocity. The charge exchange frequency is defined by:

$$
\beta_{i}(x, \mathbf{v})=\int d \mathbf{w}|\mathbf{v}-\mathbf{w}| \sigma_{c e}(|\mathbf{v}-\mathbf{w}|) f_{i}(x, \mathbf{w}) .
$$

Notice that the charge exchange cross section is only a function of the relative speed $v_{r} \equiv\left|\mathbf{v}_{H}-\mathbf{v}_{p}\right|$. Now we consider only the stationary solution, 
hence $\partial f_{i} / \partial t=0$. From the integration of first, second and third moments of Eq. (3) we get the fluid equations for neutral hydrogen:

$$
\begin{array}{r}
\frac{\partial}{\partial x}\left[\rho_{H} u_{H}\right]=0, \\
\frac{\partial}{\partial x}\left[\rho_{H} u_{H}^{2}+P_{H}\right]=-q_{m}, \\
\frac{\partial}{\partial x}\left[\frac{1}{2} \rho_{H} u_{H}^{3}+\frac{\gamma}{\gamma-1} P_{H} u_{H}\right]=-q_{e} .
\end{array}
$$

The term $q_{m}$ and $q_{e}$ are the flux of momentum and energy which are transferred from neutral hydrogen to protons, i.e:

$$
\begin{array}{r}
q_{m}=\int d v_{H} d v_{p}\left(v_{H}-v_{p}\right) \sigma_{c e}\left(v_{r}\right) v_{r} f_{H}\left(v_{H}\right) f_{p}\left(v_{p}\right) \\
q_{e}=\int d v_{H} d v_{p} \frac{1}{2}\left(v_{H}^{2}-v_{p}^{2}\right) \sigma_{c e}\left(v_{r}\right) v_{r} f_{H}\left(v_{H}\right) f_{p}\left(v_{p}\right) .
\end{array}
$$

In order to get an analytic expression for $q_{m}$ and $q_{e}$ we follow Ref. 9. First of all we assume that both hydrogen and protons distributions are Maxwellian, with a bulk velocity $\mathbf{u}_{i}(x)$, along the $x$ direction, and a temperature $T_{i}(x)$, i.e. $f_{i}(x, \mathbf{v})=\rho_{i}\left(\pi v_{T_{i}}^{2}\right)^{-3 / 2} e^{-\left(\mathbf{v}-\mathbf{u}_{i}\right)^{2} / v_{T_{i}}^{2}}$, where the thermal speed is defined as $v_{T_{i}}=\sqrt{2 P_{i} / \rho_{i}}$. Moreover we note that for $v_{r} \lesssim 2000 \mathrm{~km} / \mathrm{s}$, the charge exchange cross section decreases only logarithmically with increasing $v_{r}$, hence we can approximate $\sigma_{c e}$ as a constant evaluated at the average relative speed and pull it out of the integrals. Under this assumptions momentum and energy transfer can be expressed as follows:

$$
\begin{array}{r}
q_{m}=\sigma_{c e}(\bar{u}) \rho_{p} \rho_{H}\left(u_{H}-u_{p}\right)\left[\bar{u}+v_{T_{H}} / h_{1}(H, p)+v_{T_{p}} / h_{1}(H, p)\right] ;(10 \\
q_{e}=\sigma_{c e}(\bar{u}) \rho_{p} \rho_{H} \frac{1}{2}\left\{\bar{u}\left(u_{H}^{2}-u_{p}^{2}\right)+\frac{3}{2}\left[v_{T_{H}}^{2} h_{2}(p, H)-v_{T_{p}}^{2} h_{2}(H, p)\right]+\right. \\
\left.+2\left(u_{H}-u_{p}\right)\left[\frac{u_{H} v_{T_{H}}^{2}}{h_{1}(p, H)}+\frac{u_{p} v_{T_{p}}^{2}}{h_{1}(H, p)}\right]\right\}
\end{array}
$$

where the average relative speed $\bar{u}$, and the functions $h_{1,2}$ are defined as:

$$
\begin{array}{r}
\bar{u}=\left[\frac{4}{\pi}\left(v_{T_{i}}^{2}+v_{T_{j}}^{2}\right)+\left(u_{i}-u_{j}\right)^{2}\right]^{1 / 2} \\
h_{1}(i, j)=\left[4\left(\frac{4}{\pi} v_{T_{i}}^{2}+\left(u_{i}-u_{j}\right)^{2}\right)+\frac{9 \pi}{4} v_{T_{n}}^{2}\right]^{1 / 2} \\
h_{2}(i, j)=\left[\frac{4}{\pi} v_{T_{i}}^{2}+\frac{64}{9 \pi} v_{T_{j}}^{2}+\left(u_{i}-u_{j}\right)^{2}\right]^{1 / 2} .
\end{array}
$$


The fluid equations for protons are similar to those for hydrogen, but include the contribution due to CRs. Following Ref. 8 it is easy to show that the flux conservation of mass, momentum and energy are:

$$
\begin{array}{r}
\frac{\partial}{\partial x}\left[\rho_{p} u_{p}\right]=0 \\
\frac{\partial}{\partial x}\left[\rho_{p} u_{p}^{2}+P_{p}+P_{c}\right]=q_{m} \\
\frac{\partial}{\partial x}\left[\frac{1}{2} \rho_{p} u_{p}^{3}+\frac{\gamma}{\gamma-1} P_{p} u_{p}\right]=-u_{p} \frac{\partial P_{c}}{\partial x}+q_{e} .
\end{array}
$$

where $P_{c}$ is the $\mathrm{CR}$ pressure which is connected to the CR distribution function through $P_{c}(x)=4 \pi / 3 \int p^{2} d p p v f_{c}(x, p)$. Equations (5)-(7) and (15)-(17) are coupled through $q_{m}$ and $q_{e}$ and can be solved numerically to get the pressures and the bulk velocities of both protons and hydrogen as implicit functions of $P_{c}$. In order to close the system of equations we need to solve also the transport equation for the CR distribution function, $f_{c}$. All the system can be solved using iterative techniques similar to those used in Ref. 8. We will describe the full procedure in a more detailed work.

It is worth stressing that the technique used allows one to take into account other important effects in the shock acceleration theory, like magnetic field amplification and turbulent heating. For the sake of simplicity here we neglect such complications in order to isolate the role of charge exchange.

\section{Results and Conclusions}

We consider a typical case of shock with the following parameters: shock speed $u_{0}=2000 \mathrm{~km} / \mathrm{s}$, total upstream density $\rho_{0}=1 \mathrm{~cm}^{-3}$, neutral fraction $50 \%$, upstream temperature $T_{0}=10^{4} \mathrm{~K}$, upstream magnetic field strength $B_{1}=10 \mu \mathrm{G}$ and maximum momentum of accelerated protons fixed to $p_{\max }=10^{4} m_{p} c$. The parameter which regulates the injection efficiency is taken $\xi_{\text {inj }}=3.95 .{ }^{8}$ For these values we get $L_{\text {prec }} / L_{c e} \simeq 150$, hence we do expect an efficient role of charge exchange, while $L_{\text {prec }} / L_{\text {ion }} \simeq 10^{-4}$ hence the ionization can be neglected as stated above. In Fig. 1 we show the velocity and the temperature profiles in the precursor for both protons and hydrogen. The dotted line shows the normalized CR pressure, which at the sub-shock position reaches the value $P_{c} / \rho_{0} u_{0}^{2}=0.14$, hence we are dealing with a mildly efficient shock. The upstream temperatures of protons and hydrogen reach the values of $5 \cdot 10^{5} \mathrm{~K}$ and $4.7 \cdot 10^{5} \mathrm{~K}$, respectively. Protons are slightly wormer than hydrogen because they also suffer the adiabatic 
compression due to the CR pressure. The narrow Balmer line width corresponding to the hydrogen temperature at ths subshock is $46 \mathrm{~km} / \mathrm{s}$, to be compared with $21 \mathrm{~km} / \mathrm{s}$ resulting in the absence af a CR precursor. The downstream temperature of shocked ions is $6.7 \cdot 10^{7} \mathrm{~K}$, which produces a broad Balmer line with a width of $1774 \mathrm{~km} / \mathrm{s}$; in the absence of acceleration the same width would be $2039 \mathrm{~km} / \mathrm{s}$. As already pointed out, this difference arises because when the acceleration is efficient a non negligible fraction of the total shock kinetic energy is channelled into CRs rather than into thermal energy.

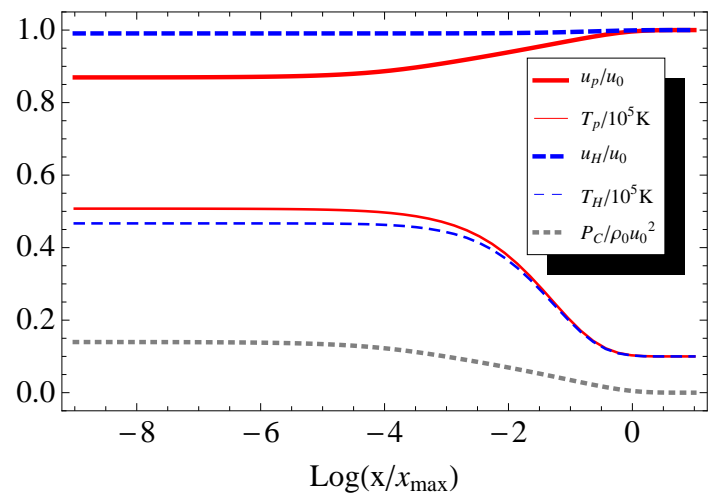

Fig. 1. Profiles of velocities and temperatures of hydrogen and protons in the precursor upstream of the shock. The thick-dotted line shows the CR pressure. All quantities are normalized as shown in the legend. The typical precursor length is $x_{\max }=D\left(p_{\max }\right) / u_{0}$.

\section{References}

1. G. Morlino, E. Amato, and P. Blasi, MNRAS, 392, 240 (2009)

2. R. A. Chevalier, R. P. Kirshner, and J. C. Raymond, ApJ, 235, 186 (1980)

3. K. Heng, PASA, 27, 23 (2009)

4. J. J. Lee, J. C. Raymond, S. Park, W. P. Blair, P. Ghavamian, P. F. Winkler, and K. Korreck, ApJ, 715, L146 (2010).

5. J. Sollerman, P. Ghavamian, P. Lundqvist, R. C. Smith, A\& A, 407, 249 (2003)

6. E. A. Helder, J. Vink, C. G. Bassa, A. Bamba, J. A. M. Bleeker, S. Funk, P. Ghavamian, K. J. van der Heyden, F. Verbunt, R. Yamazaki, Science, 325, $719(2009)$

7. E. A. Helder, D. Kosenko, J. Vink, ApJ, 719L, 140 (2010)

8. E. Amato, and P. Blasi, MNRAS, 371, 1251 (2006)

9. H. L. Pauls, G. P. Zank, and L. L.Williams, Jour. of Geophys. Research, 100, $21(1995)$ 